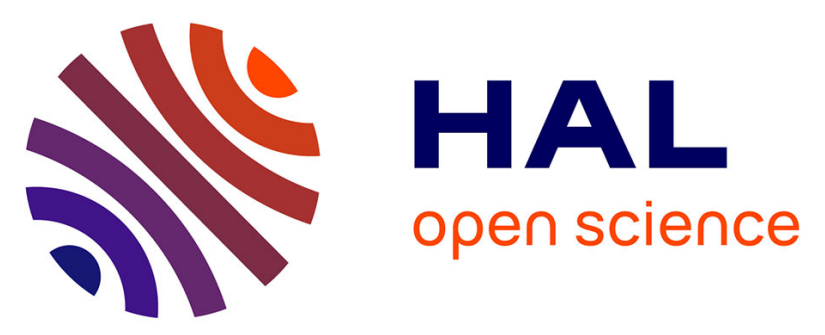

\title{
Interaction entre différentes souches de Rhizobium lupini et les espèces ou cultivars de lupin (Lupinus albus, Lupinus luteus et Lupinus mutabilis)
}

Bernard Lagacherie, Michèle Bours, Jean-Jacques Giraud, Georges Sommer

\section{- To cite this version:}

Bernard Lagacherie, Michèle Bours, Jean-Jacques Giraud, Georges Sommer. Interaction entre différentes souches de Rhizobium lupini et les espèces ou cultivars de lupin (Lupinus albus, Lupinus luteus et Lupinus mutabilis). Agronomie, 1983, 3 (8), pp.809-816. hal-00884574

\section{HAL Id: hal-00884574 https://hal.science/hal-00884574}

Submitted on 1 Jan 1983

HAL is a multi-disciplinary open access archive for the deposit and dissemination of scientific research documents, whether they are published or not. The documents may come from teaching and research institutions in France or abroad, or from public or private research centers.
L'archive ouverte pluridisciplinaire HAL, est destinée au dépôt et à la diffusion de documents scientifiques de niveau recherche, publiés ou non, émanant des établissements d'enseignement et de recherche français ou étrangers, des laboratoires publics ou privés. 


\title{
Interaction entre différentes souches de Rhizobium lupini et les espèces ou cultivars de lupin (Lupinus albus, Lupinus luteus et Lupinus mutabilis)
}

\author{
Bernard LAGACHERIE, Michèle BOURS, Jean-Jacques GIRAUD \& Georges SOMMER \\ I.N.R.A., Laboratoire de Microbiologie des sols, BV 1540, F 21034 Dijon Cedex
}

RÉSUMÉ

\begin{abstract}
L'efficacité fixatrice de souches de Rhizobium lupini a été mesurée en serre sur Lupinus albus, luteus et mutabilis. De fortes interactions se manifestent : 86 p. 100 des souches testées ont une bonne efficacité sur L. albus, 52 p. 100 sur L. mutabilis et 40 p. 100 sur L. luteus; 22 p. 100 des souches ont une bonne efficacité fixatrice sur les trois espèces. Les interactions observées en serre se conservent au champ où les souches fixatrices augmentent le rendement en grain et la teneur en protéines des grains.

Il apparaît aussi des interactions entre cultivars de $L$. albus et souches de $R$. lupini.
\end{abstract}

Mots clés additionnels : Fixation d'azote, inoculum, inoculation, symbiose.

Interaction between Rhizobium lupini strains and species or cultivars of lupin (Lupinus albus, Lupinus luteus and Lupinus mutabilis).

Efficiency of Rhizobium lupini strains was evaluated in the greenhouse on Lupinus albus, L. luteus and L. mutabilis. Strong species $\times$ strain interactions were observed: $86 \%$ of strains were fully effective on L. albus, $52 \%$ on $L$. mutabilis and $40 \%$ on $L$. luteus $; 22 \%$ of strains were very effective on all three species. Species $\times$ strain interactions persisted in a field experiment where effective strains increased yield and protein level of grain.

Interactions between $L$. albus cultivars and $R$. lupini strains were also observed.

Additional key words : Nitrogen fixation, inoculum, inoculation, symbiosis.

\section{INTRODUCTION}

Une étude précédente (LAGACHERIE et al., 1976) a montré que Rhizobium lupini (Schroet.) Eckh et al., bactérie provoquant la formation de nodosités sur les racines des genres Lupinus et Ornithopus (GRAHAM, 1976), est fréquemment absent des sols de culture français; il est nécessaire, dans ces sols, d'inoculer les semences de lupin avec des souches de Rhizobium fixatrices.

Les premières souches de $R$. lupini isolées lors de cette étude se sont révélées d'efficacité fixatrice d'azote variable sur Lupinus albus L., L. luteus L. et L. angustifolius L. La variabilité de l'efficacité fixatrice des souches a été observée par LANGE \& PARKER (1960) sur L. albus, L. angustifolius et $L$. digitatus Forsk., par CARADUS \& SILVESTER (1979) sur $L$. arboreus Sims. De plus des interactions espèce $\times$ souche se manifestent pour ce caractère (LAGACHERIE et al., 1976).

Plusieurs espèces annuelles de lupin peuvent être cultivées (GLADSTONE, 1970) et 3 au moins présentent un intérêt agronomique en France (LENOBLE \& PICARD, 1980):
L. albus, L. luteus et L. mutabilis Sweet. Il est donc indispensable de déterminer l'efficacité fixatrice des souches de $R$. lupini sur ces espèces, en tenant compte éventuellement de l'influence du cultivar au sein de l'espèce ; les souches retenues pour la fabrication d'inoculum devront être polyvalentes.

Nous avons testé un lot de 41 souches isolées de sols français sur les 3 espèces $L$. albus, $L$. luteus et $L$. mutabilis et sur 8 cultivars de $L$. albus, espèce la plus productive. Ce travail a été conduit en serre et au champ.

\section{MATÉRIEL ET MÉTHODES}

\section{A. Isolement des souches}

Les souches de $R$. lupini testées dans les différents essais ont été isolées dans des échantillons de sols agricoles français en utilisant comme plante-piège soit L. albus (souches LB) soit L. luteus (souches LL). 
Dans un pot de matière plastique d' 11 , rempli d' $1 / 21$ de sable stérilisé (sable extra siliceux B4, Silices et Réfractaires de la Méditerranée, Marseille), $100 \mathrm{~g}$ de la terre considérée sont étalés, puis recouverts d'une couche de $4 \mathrm{~cm}$ de sable stérilisé. Quatre graines de lupin, désinfectées par trempage pendant $30 \mathrm{mn}$ dans une solution d'hypochlorite de calcium ( $70 \mathrm{~g}$ d'hypochlorite de calcium $115-120^{\circ}$ chlorométriques dans $1000 \mathrm{~cm}^{3}$ d'eau, agités pendant $30 \mathrm{mn}$ et filtrés) et rincées à l'eau stérile, sont semées dans chaque pot; la solution d'arrosage n'apporte pas d'azote (LAGACHERIE et al., 1977).

Au stade floraison, plusieurs nodosités ont été prélevées dans chaque pot, désinfectées extérieurement par tempage dans une solution aqueuse de $\mathrm{HgCl}_{3}$ à 1 p. 1000 pendant $5 \mathrm{mn}$ puis rincées abondamment à l'eau stérile ; une suspension de Rhizobium, réalisée par écrasement d'une nodosité dans de l'eau stérile a été étalée sur milieu nutritif gélosé de BERGERSEN (1961) complété par 0,2 g/l d'extrait de levure, à la dilution permettant d'obtenir des colonies isolées; 3 repiquages successifs ont été effectués pour obtenir une culture pure; seules les souches à croissance lente (une par sol) ont été retenues : il s'agit des souches pour lesquelles des colonies apparaissent après $5 \mathrm{j}$ au moins de croissance, à $28^{\circ} \mathrm{C}$, sur milieu nutritif gélosé.

\section{B. Croissance des végétaux en serre}

Les plantes sont cultivées dans des pots en matière plastique d'une contenance de $51 ; 11$ de graviers assure le drainage au fond du pot; le support de culture était de la perlite (Perlisol 40, la Vermiculite et la Perlite, Nanterre). Après levée des graines, désinfectées au semis comme précédemment, 3 plantes sont conservées et inoculées par une suspension aqueuse d'une culture de Rhizobium sur milieu nutritif gélosé apportant au minimum $5.10^{9}$ bactéries par pot. Pendant la croissance des plantes, l'apport de la solution nutritive mentionnée ci-dessus est réalisé automatiquement, en fonction de l'évapotranspiration d'un pot témoin.

Dans chacun des essais dont les caractéristiques sont regroupées dans le tableau 1 , les différentes souches sont réparties sur une espèce selon un dispositif en blocs randomisés.

La récolte a été effectuée à la fin de la floraison du $1^{\text {er }}$ étage floral. Le poids $\sec$ des parties aériennes a servi de mesure de l'efficacité fixatrice des souches; l'examen des racines a montré que toutes les souches utilisées avaient provoqué une nodulation.

Une analyse de variance effectuée sur les résultats de chaque espèce (ou cultivar) d'un essai permet de tester la signification (test F) du facteur souche, puis, sur la totalité de l'essai, la signification de l'interaction espèce (ou cultivar) $\times$ souche. Les souches sont différenciées sur chaque espèce (ou cultivar) par le test de DunCAN au seuil de probabilité de 5 p. 100.

Pour permettre une comparaison entre espèces et entre essais, les résultats ont été exprimés en fonction du rendement moyen (base 100) des 2 souches LL13 et LB29 communes à tous les essais. Nous avons défini les classes d'efficacité fixatrice par rapport à cette base 100 : souches peu ou pas fixatrices entre 0 et 40 , souches moyennement fixatrices entre 40 et 80 et souches bonnes fixatrices audessus de 80 .

\section{Essai au champ}

Un essai au champ a été mis en place au domaine I.N.R.A. de Dijon-Epoisses en 1978.

Quatre souches de $R$. lupini et un témoin non inoculé ont été comparés sur les 3 espèces L. albus cv. "Blanca", L. luteus cv. "Sulfa» et L. mutabilis cv. «LM13». Dans chaque espèce, les traitements sont répartis en 6 blocs randomisés. Chaque parcelle de $7 \mathrm{~m}$ de long comporte 6 rangs écartés de $33 \mathrm{~cm}$.

Les graines ont été inoculées par un inoculum-tourbe de façon à obtenir une richesse d'au moins $10^{6}$ Rhizobium/graine. Le semis a été réalisé le 13 mars après inoculation et ressuyage des graines, à l'aide d'un semoir à main EBRA type SJ5; un flambage du semoir à l'alcool a été effectué à chaque changement de souche. Le peuplement était de l'ordre de 70 plantes par $\mathrm{m}^{2}$ pour $L$. albus et L. mutabilis et de 40 pour L. luteus.

La récolte a été faite par microbatteuse sur les 4 rangs centraux. La quantité de grain sec a été mesurée et le pourcentage de protéines déterminé par l'analyseur PROMETER type MKII.

Les dates de récolte se sont échelonnées en fonction de la précocité des espèces: L. luteus le 23 août, L. albus le 16 septembre et $L$. mutabilis le 30 septembre.

TABLEAU 1

Caractéristiques des essais en serre.

Characteristics of the greenhouse experiments.

\begin{tabular}{|c|c|c|c|c|c|}
\hline \multirow{2}{*}{$N^{\circ}$ d'essai } & \multicolumn{2}{|c|}{ Végétation } & \multirow{2}{*}{$\begin{array}{l}\text { Nombre de } \\
\text { répétitions }\end{array}$} & \multirow{2}{*}{ Espèces } & \multirow{2}{*}{$\begin{array}{c}\text { Nombre de } \\
\text { souches }\end{array}$} \\
\hline & Période & Durée (semaines) & & & \\
\hline I & Printemps 77 & 12 & 3 & L. albus $\left({ }^{1}\right)$ L. luteus $\left({ }^{2}\right)$ & $17(*)$ \\
\hline II & Automne 77 & 11 & 3 & L. albus $\left({ }^{1}\right)$ L. luteus $\left({ }^{2}\right)$ & $18(*)$ \\
\hline III & Hiver 78 & 13 & 3 & L. albus $\left({ }^{1}\right)$ L. mutabilis $\left({ }^{3}\right)$ & $21\left(^{*}\right)$ \\
\hline IV & Automne 79 & 12 & 4 & L. albus $\left({ }^{1}\right) \quad$ L. luteus $\left({ }^{2}\right)$ L. mutabilis $\left({ }^{3}\right)$ & $11(*)$ \\
\hline $\mathrm{V}$ & Printemps 79 & 10 & 4 & L. albus (8 cultivars) $\left(^{4}\right)$ & 6 \\
\hline
\end{tabular}

$\left(^{*}\right)$ Comparées à un témoin non inoculé.

$\left({ }^{1}\right)$ : L. albus cv «Blanca ; $\left({ }^{2}\right):$ L. luteus cv «Sulfa »; $\left({ }^{3}\right):$ L. mutabilis cv «LM13»; $\left({ }^{4}\right)$ : «Blanca », « Kali », « Kalina », «La57 », "Lublanc », «Multolupa », "Neuland » et "Ultra». 


\section{RF́SULTATS}

\section{A. Essais sur L. albus et luteus (I et II)}

Un lot de 32 souches de $R$. lupini a été testé en serre lors de 2 essais sur les 2 espèces L. albus cv. "Blanca" et L. luteus cv. «Sulfa ». Un témoin non inoculé et 17 souches ont été comparées dans l'essai I, un témoin non inoculé, 3 souches de l'essai I (LL13, LL19 et LB29) et 15 nouveaux isolements dans l'essai II.

Les résultats des 2 essais sont regroupés dans le tableau 2. Compte tenu de la période de végétation moins favorable et d'une mise à fleur plus précoce, la croissance des plantes a été moindre lors de l'essai II, surtout pour L. albus.

Dans les 2 essais, l'effet souche est significatif au seuil de probabilité de 1 p. 100 sur chaque espèce : les souches de $R$. lupini sont d'efficacité variable.

Sur L. albus, la souche LL90 (essai I) est non fixatrice ; 14 souches (soit 82 p. 100 du total) de l'essai I et 13 souches (72 p. 100) de l'essai II sont bonnes fixatrices. Sur $L$. luteus, les souches LB86, LL88, LL97 et LB822 (essai I), LL90 et LL110 (essai II) sont très peu fixatrices; 6 souches (35 p. 100) de l'essai I et 9 souches $(50$ p. 100) de l'essai II sont bonnes fixatrices. La répartition des souches sur une espèce selon leur efficacité est comparable dans chaque essai, mais elle diffère d'une espèce à l'autre. Certaines souches sont d'efficacité faible (LB86, LL90), moyenne (LL96, LL101) ou bonne (LL19, LB29, LL13, ...) sur les 2 espèces alors que d'autres souches (LL84, LL89, LB822, LL1052) sont bonnes fixatrices sur L. albus et faibles sur L. luteus ou inversement (souche LL15) faible sur L. albus et bonne sur L. luteus. L'existence d'une interaction espèce $\times$ souche significative dans chaque essai au seuil de probabilité de 1 p. 100 confirme statistiquement ces différences de comportement.

\section{B. Essai sur L. albus et mutabilis (III)}

13 souches déjà testées, 8 nouvelles souches ainsi qu'un témoin non inoculé ont été comparés en serre sur $L$. albus $\mathrm{cv}$ « Blanca » et $L$. mutabilis cv « LM13». Les résultats sont reportés dans le tableau 3.

Sur chaque espèce, l'effet souche est significatif au seuil de probabilité de 1 p. 100 : les souches sont d'efficacité fixatrice variable. La souche LL90 est inefficace sur les 2 espèces.

Mais alors que, sur L. albus, les 20 autres souches sont bonnes fixatrices, sur L. mutabilis, 12 souches seulement (soit 57 p. 100 du total) le sont; le comportement des souches n'est pas le même sur les 2 espèces, les souches LL96, LLH25, LB28, LL94 et LLH31 sont bonnes fixatrices sur $L$. albus et moyennes ou peu fixatrices sur L. mutabilis ; parmi elles, LL96, LB28 et LL94 étaient bonnes fixatrices sur $L$. luteus. Statistiquement, ces différences de comportement se traduisent par une interaction espèce $\times$ souches significative au seuil de probabilité de 1 p. 100.

Les souches déjà testées conservent l'efficacité fixatrice mesurée dans les essais précédents, à l'exception des deux souches LL82 et LL96.

\section{Essais sur L. albus, luteus et mutabilis}

\section{Essai en serre (IV)}

Dix souches choisies parmi celles déjà testées, une nouvelle souche et un témoin non inoculé ont été comparés sur les 3 espèces L. albus cv «Blanca», L. luteus cv

\section{TABLEAU 2}

Efficacité fixatrice de 17 souches (essai I) et 18 souches (essai II) de $\mathrm{R}$. lupini associées à L. albus et L. luteus (poids sec des parties aériennes en p. 100 de la moyenne des souches LL13 et LB29; moyenne de 3 répétitions).

Efficiency of 17 (essai I) and 18 (essai II) R. lupini strains growing with $\mathrm{L}$. albus and $\mathrm{L}$. luteus plants (dry weight \% of the average of strains $L L 13$ and LB29; mean of 3 replicates).

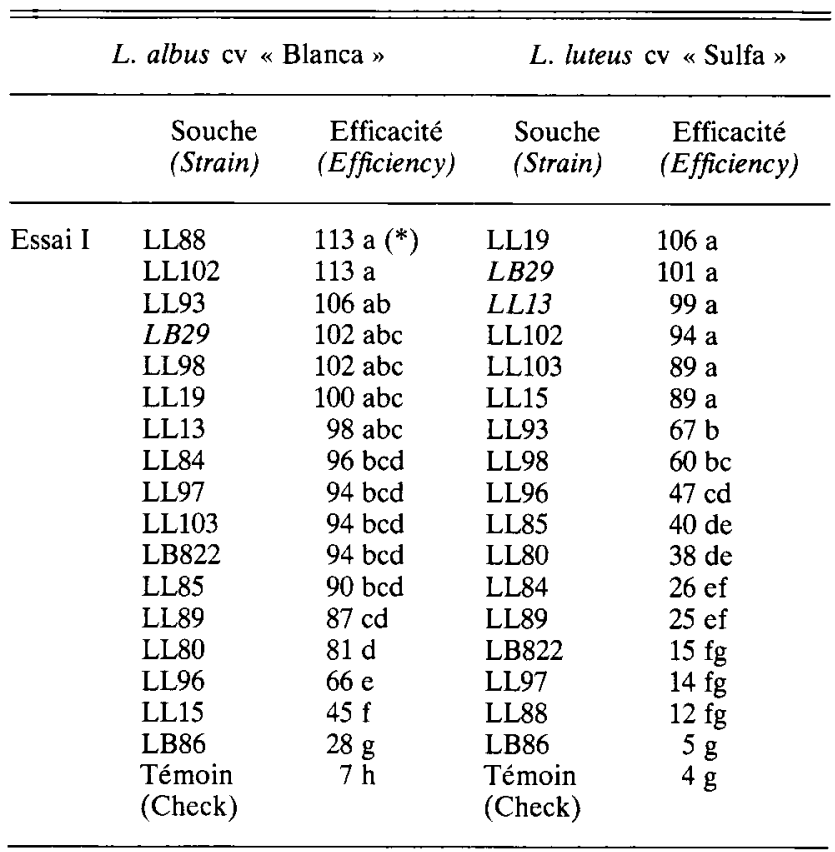

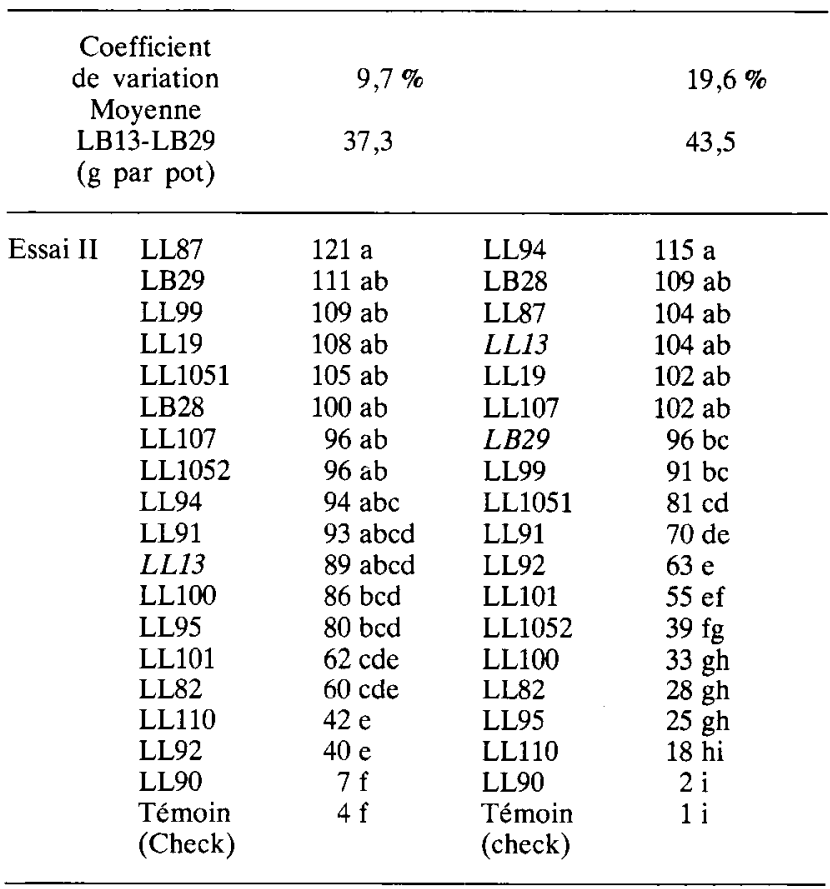

Coefficient

de variation

Moyenne

LL13-LB29

$20,0 \%$

$12,5 \%$

(g/pot)

34,8

(*) Les valeurs suivies de la (ou des) même lettre ne diffèrent pas au seuil de probabilité de $5 \mathrm{p} .100$ (test de DUNCAN).

«Sulfa» et L. mutabilis cv « LM13». Les résultats sont reportés dans le tableau 4.

Sur chaque espèce l'effet souche est significatif au seuil de probabilité de 1 p. 100 . L'interaction espèce $\times$ souche, 


\section{TABLEAU 3}

Efficacité fixatrice de 21 souches de $\mathrm{R}$. lupini associées à $\mathrm{L}$. albus et L. mutabilis (poids sec des parties aériennes en p. 100 de la moyenne des souches LL13 et LB29; moyenne de 3 répétitions).

Efficiency of 21 R. lupini strains with L. albus and L. mutabilis (shoot dry weight as \% of the average of strains LL13 and LB29; mean of 3 replicates).

\begin{tabular}{|c|c|c|c|}
\hline \multicolumn{2}{|c|}{ L. albus cv « Blanca» } & \multicolumn{2}{|c|}{ L. mutabilis cv "LM13» } \\
\hline $\begin{array}{l}\text { Souche } \\
\text { (Strain) }\end{array}$ & $\begin{array}{c}\text { Efficacité } \\
\text { (Efficiency) }\end{array}$ & $\begin{array}{l}\text { Souche } \\
\text { (Strain) }\end{array}$ & $\begin{array}{c}\text { Efficacité } \\
\text { (Efficiency) }\end{array}$ \\
\hline LL113 & $115 \mathrm{a}$ & $L L 13$ & $113 \mathrm{a}$ \\
\hline LL1051 & $110 \mathrm{ab}$ & LL87 & $112 \mathrm{a}$ \\
\hline LL94 & $109 a b$ & LLH26 & $111 \mathrm{ab}$ \\
\hline LLH31 & $108 \mathrm{ab}$ & LL84 & $110 a b c$ \\
\hline LLH26 & $106 \mathrm{ab}$ & LL1051 & $98 \mathrm{abcd}$ \\
\hline LLH25 & $106 \mathrm{ab}$ & LLH40 & 95 abcde \\
\hline$L B 29$ & $105 \mathrm{ab}$ & LB822 & 94 bcde \\
\hline LB28 & $100 \mathrm{ab}$ & LL82 & 93 bcde \\
\hline LL82 & $100 \mathrm{ab}$ & LL88 & 92 cde \\
\hline LL84 & $99 \mathrm{ab}$ & LB84 & $92 \mathrm{de}$ \\
\hline LB821 & $98 \mathrm{ab}$ & LLH8 & $88 \mathrm{def}$ \\
\hline LLH40 & $98 \mathrm{ab}$ & $L B 29$ & $87 \mathrm{def}$ \\
\hline LL96 & $98 \mathrm{ab}$ & LB821 & $79 \mathrm{efg}$ \\
\hline LB822 & $97 \mathrm{ab}$ & LL113 & $72 \mathrm{fg}$ \\
\hline LB84 & $96 \mathrm{ab}$ & LL1052 & $67 \mathrm{gh}$ \\
\hline$L L 13$ & $95 \mathrm{ab}$ & LL96 & $52 \mathrm{hi}$ \\
\hline LL87 & $95 \mathrm{ab}$ & LLH25 & $51 \mathrm{i}$ \\
\hline LL88 & $94 \mathrm{~b}$ & LB28 & $43 \mathrm{ij}$ \\
\hline LL1052 & $92 \mathrm{~b}$ & LL94 & $31 \mathrm{jk}$ \\
\hline LLH8 & $90 \mathrm{~b}$ & LLH31 & $23 \mathrm{k}$ \\
\hline LL90 & $19 \mathrm{c}$ & LL90 & 61 \\
\hline $\begin{array}{l}\text { Témoin } \\
\text { (Check) }\end{array}$ & $7 c$ & $\begin{array}{l}\text { Témoin } \\
\text { (Check) }\end{array}$ & 51 \\
\hline \multirow{2}{*}{\multicolumn{2}{|c|}{$\begin{array}{c}\text { Coefficient } \\
\text { de variation } \\
\text { Moyenne LL13-LB29 } \\
(\mathrm{g} \text { par pot) }\end{array}$}} & $11,0 \%$ & $13,7 \%$ \\
\hline & & 37,4 & 27,8 \\
\hline
\end{tabular}

significative au seuil de probabilité de 1 p. 100 , permet de mettre en évidence différents comportements de souches: 2 souches (LL13 et LB29) sont bonnes fixatrices sur les 3 espèces ; les souches LL82, LB822, LL88 et LL113 ne sont bonnes fixatrices que sur L. albus et mutabilis et peu fixatrices sur L. luteus ; la souche LLH31 est bonne fixatrice sur $L$. albus et luteus et peu fixatrice sur $L$. mutabilis; enfin la souche LL15 est bonne fixatrice sur $L$. luteus et peu fixatrice sur les 2 autres espèces. Les 3 dernières souches se rapprochent de l'un de ces comportements: les souches LB28 et LL96 sont proches de LLH31, la souche LB26 proche de LL82.

\section{Essai au champ}

Quatre souches bien typées dans les essais précédents ainsi qu'un témoin non inoculé ont été comparés sur les 3 espèces: LL13 et LB29, bonnes fixatrices sur les 3 espèces; LB822, bonne fixatrice sur L. albus et mutabilis et LB86, peu fixatrice sur L. albus et luteus et non testée sur L. mutabilis. Les rendements en grain sec et la teneur en protéines de ces grains sont regroupés dans le tableau 5.

Dans chaque espèce l'effet souche sur la récolte de grain est significatif au seuil de probabilité de 1 p. 100 ; l'interaction espèce $\times$ souche l'est aussi. Les souches se différencient et se classent au champ de la même facon qu'en serre. La souche LB86 se révèle également peu fixatrice sur L. mutabilis.

Sur la teneur en protéines des grains, l'effet souche est significatif au seuil de probabilité de 1 p. 100 : les souches fixatrices entraînent sur $L$. albus et luteus une augmentation de cette teneur par rapport au témoin non inoculé, les souches non fixatrices restant à son niveau ; sur L. mutabilis, la souche LL13 est supérieure au témoin non inoculé, LL29 lui est comparable et les souches LB822 (fixatrice) et LB86 (non fixatrice) inférieures. L'interaction espèce $\times$ souche sur le rendement en protéines en est donc renforcée.

\section{TABLEAU 4}

Efficacité fixatrice de 11 souches de $\mathrm{R}$. lupini associées à $\mathrm{L}$. albus, L. luteus et $\mathrm{L}$. mutabilis. (poids sec des parties aériennes en p. 100 de la moyenne des souches $L L 13$ et LB29; moyenne de 4 répétitions).

Efficiency of 11 R. lupini strains with L. albus, L. luteus and L. mutabilis (shoot dry weight as \% of the average of strains LL13 and L879: mean of 4 replicates).

\begin{tabular}{|c|c|c|c|c|c|}
\hline \multicolumn{2}{|c|}{ L. albus cv «Blanca » } & \multicolumn{2}{|c|}{ L. luteus cv «Sulfa» } & \multicolumn{2}{|c|}{ L. mutabilis cv « LM13" } \\
\hline $\begin{array}{l}\text { Souche } \\
\text { (Strain) }\end{array}$ & $\begin{array}{l}\text { Efficacité } \\
(\text { Efficiency) }\end{array}$ & $\begin{array}{l}\text { Souche } \\
\text { (Strain) }\end{array}$ & $\begin{array}{c}\text { Efficacité } \\
\text { (Efficiency) }\end{array}$ & $\begin{array}{l}\text { Souche } \\
\text { (Strain) }\end{array}$ & $\begin{array}{c}\text { Efficacité } \\
\text { (Efficiency) }\end{array}$ \\
\hline $\begin{array}{l}\text { LB29 } \\
\text { LLH31 } \\
\text { LB28 } \\
\text { LB822 } \\
\text { LB26 } \\
\text { LL88 } \\
\text { LL13 } \\
\text { LL96 } \\
\text { LL113 } \\
\text { LL82 } \\
\text { LL15 } \\
\text { Témoin } \\
\text { (Check) }\end{array}$ & $\begin{array}{c}107 \mathrm{a} \\
102 \mathrm{ab} \\
99 \mathrm{ab} \\
98 \mathrm{ab} \\
94 \mathrm{ab} \\
93 \mathrm{ab} \\
93 \mathrm{ab} \\
92 \mathrm{ab} \\
90 \mathrm{~b} \\
90 \mathrm{~b} \\
34 \mathrm{c} \\
6 \mathrm{~d}\end{array}$ & $\begin{array}{l}\text { LL15 } \\
L L 13 \\
L B 29 \\
\text { LB28 } \\
\text { LLH31 } \\
\text { LL96 } \\
\text { LB822 } \\
\text { LL82 } \\
\text { LB26 } \\
\text { LL113 } \\
\text { LL88 } \\
\text { Témoin } \\
\text { (Check) }\end{array}$ & $\begin{array}{c}104 \mathrm{a} \\
102 \mathrm{ab} \\
98 \mathrm{ab} \\
94 \mathrm{bc} \\
93 \mathrm{bc} \\
92 \mathrm{c} \\
34 \mathrm{~d} \\
23 \mathrm{e} \\
21 \mathrm{e} \\
16 \mathrm{e} \\
15 \mathrm{e} \\
4 \mathrm{f}\end{array}$ & $\begin{array}{l}\text { LL13 } \\
L B 29 \\
\text { LL88 } \\
\text { LL82 } \\
\text { LL113 } \\
\text { LB822 } \\
\text { LB26 } \\
\text { LL96 } \\
\text { LB28 } \\
\text { LLH31 } \\
\text { LL15 } \\
\text { Témoin } \\
\text { (Check) }\end{array}$ & $\begin{array}{c}102 \mathrm{a} \\
98 \mathrm{a} \\
91 \mathrm{ab} \\
91 \mathrm{ab} \\
86 \mathrm{ab} \\
81 \mathrm{~b} \\
75 \mathrm{~b} \\
55 \mathrm{c} \\
46 \mathrm{c} \\
10 \mathrm{~d} \\
9 \mathrm{~d} \\
7 \mathrm{~d}\end{array}$ \\
\hline \multicolumn{2}{|c|}{$\begin{array}{l}\text { Coefficient de variation } \\
\text { Moyenne LL13-LB29 } \\
\text { (g par pot) }\end{array}$} & $\begin{array}{l}10,9 \% \\
38,2\end{array}$ & $\begin{array}{l}10,1 \% \\
47,7\end{array}$ & & $\begin{array}{l}16,9 \% \\
30,3\end{array}$ \\
\hline
\end{tabular}


TABLEAU 5

Production de grains sec et teneur en protéines de $\mathrm{L}$. albus, L. luteus et $\mathrm{L}$. mutabilis associés au champ à 4 souches de $\mathrm{R}$. lupini ; moyenne de 6 répétitions.

Grain yield and protein level of L. albus, L. luteus and L. mutabilis plants growing with 4 R. lupini strains; mean of 6 replicates.
L. albus $\mathrm{cv}$ « Blanca »
L. luteus $\mathrm{cv}$ «Sulfa»
L. mutabilis cv « LM13»

\begin{tabular}{|c|c|c|c|c|c|c|}
\hline \multirow{2}{*}{ Souches } & \multicolumn{2}{|c|}{ grain sec } & \multicolumn{2}{|c|}{ grain sec } & \multicolumn{2}{|c|}{ grain $\mathrm{sec}$} \\
\hline & $\mathrm{t} / \mathrm{ha}$ & \% protéines & t/ha & $\%$ protéines & $\mathrm{t} / \mathrm{ha}$ & $\%$ protéines \\
\hline$L B 29$ & $4,15 \mathrm{a}$ & $38,6 \mathrm{a}$ & $1,87 \mathrm{a}$ & $43,3 \mathrm{a}$ & $1,80 \mathrm{a}$ & $48,9 \mathrm{~b}$ \\
\hline$L L 13$ & $3,96 \mathrm{ab}$ & $38.2 \mathrm{a}$ & $1,82 \mathrm{a}$ & $43.4 \mathrm{a}$ & $1.80 \mathrm{a}$ & $50,2 \mathrm{a}$ \\
\hline LB822 & $3,79 \mathrm{~b}$ & $38,4 \mathrm{a}$ & $0,62 \mathrm{~b}$ & $35,5 \mathrm{~b}$ & $1,82 \mathrm{a}$ & $47,4 \mathrm{c}$ \\
\hline LB86 6 & $2.91 \mathrm{c}$ & $35,0 \mathrm{~b}$ & $0,53 \mathrm{~b}$ & $34,3 \mathrm{c}$ & $1.26 \mathrm{c}$ & $47,2 \mathrm{c}$ \\
\hline Témoin & $2,82 \mathrm{c}$ & $35,6 \mathrm{~b}$ & $0,48 \mathrm{~b}$ & $34,7 \mathrm{bc}$ & $1,39 \mathrm{~b}$ & $48,7 \mathrm{~b}$ \\
\hline $\begin{array}{l}\text { Cocfficient } \\
\text { de variation }\end{array}$ & $6,4 \%$ & $2,1 \%$ & $12,5 \%$ & $2,2 \%$ & $5,4 \%$ & $1,2 \%$ \\
\hline
\end{tabular}

\section{Synthèse des résultats}

Compte tenu de la stabilité de l'efficacité fixatrice des souches, il est possible de regrouper les résultats des différents essais.

Sur chaque espèce, les souches de $R$. lupini sont d'efficacité fixatrice variable (tabl.6), mais, sur L.albus, on obtient une plus forte proportion $(86 \mathrm{p} .100)$ de souches ayant une bonne efficacité fixatrice que sur $L$. mutabilis (52 p. 100) et que sur L. luteus (40 p. 100).

Sur les espèces prises 2 à 2 (fig. 1), les souches de $R$. lupini peuvent avoir des comportements différents: l'efficacité fixatrice est la même sur chaque espèce ou diffère selon l'espèce. Les souches dont le comportement reste identique (tabl. 7) sont proportionnellement les plus nombreuses dans la comparaison: L. albus-L. mutabilis (62 p. 100), puis L. albus-L. luteus ( 49 p. 100) et enfin L. luteus-L. mutabilis (33 p. 100). Par le jeu des interactions, et toutes les combinaisons n'ayant pas été testées, 4 souches seulement (LL13, LB29, LL87 et LL1051) ont une bonne activité fixatrice sur les 3 espèces et 2 souches (LB86 et LL90), une activité fixatrice faible ou nulle.

\section{TABLEAU 6}

Distribution, par classe d'efficacité fixatrice, des différentes souches de $\mathrm{R}$. lupini testées sur chaque espèce de lupin (en p. 100 dans chaque classe).

Distribution of all R. lupini strains tested in efficiency classes on every lupin species (\% par class).

\begin{tabular}{lcccc}
\hline \hline & $\begin{array}{c}\text { Nombre de } \\
\text { souches } \\
\text { testées }\end{array}$ & \multicolumn{4}{c}{$\begin{array}{c}\text { Classes } \\
\text { d'efficacité } \\
40-80\end{array}$} & $80-120$ \\
\hline L. albus & 41 & 7 & 7 & 86 \\
L. luteus & 35 & 46 & 14 & 40 \\
L. mutabilis & 23 & 17,5 & 26,5 & 52 \\
\hline \hline
\end{tabular}

\section{Cultivars de L. albus (Essai V)}

Cinq souches de $R$. lupini bonnes fixatrices sur $L$. albus cv «Blanca» (LL13, LB28, LB29, LL82 et LL84) et une, peu fixatrice (LL15), ont été essayées en serre sur 8 culti-
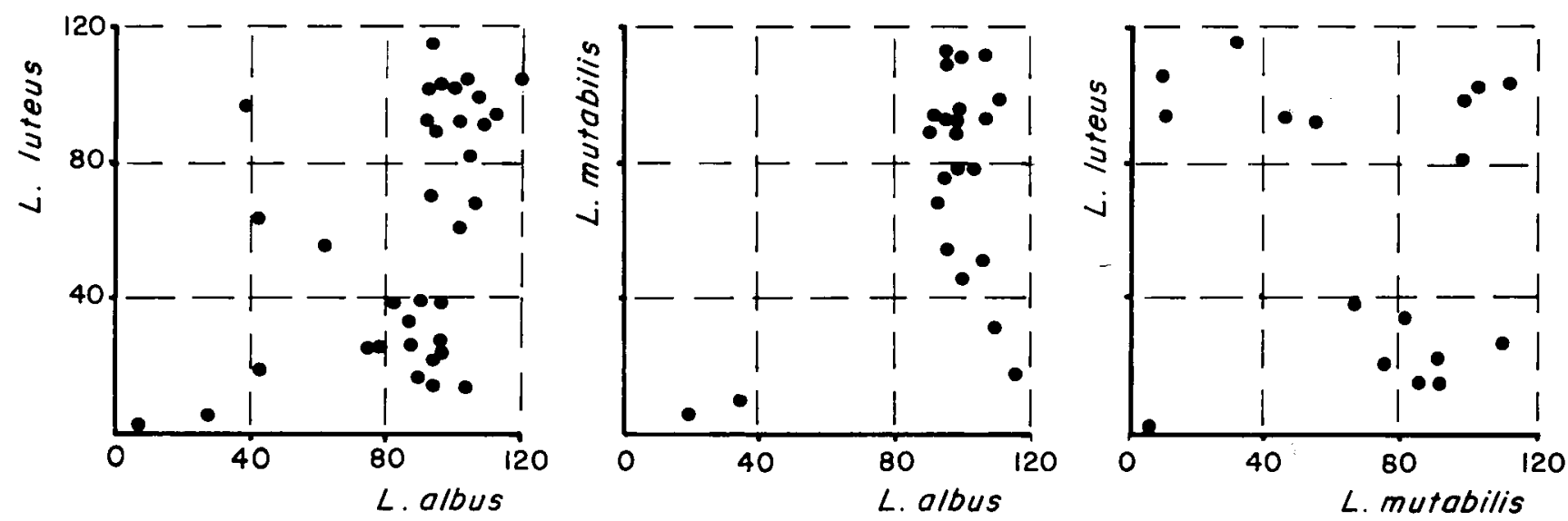

Figure 1

Efficacité comparée des souches de $\mathrm{R}$. lupini sur les 3 espèces de lupin prises deux à deux. (Abscisses et ordonnées: poids sec des parties aériennes en $p .100$ de la moyenne des souches LL13 et LB29).

Comparative effectiveness of $\mathrm{R}$. lupini strains on three lupin species taken two by two (Abscissa and ordinate: shoot dry weight as \% of the average of strains LL13 and LB29). 
TABLEAU 7

Récapitulatif des souches de R. lupini dont l'efficacité fixatrice ne varie pas lors des comparaisons sur 2 espèces de lupin (en classes d'efficacité fixatrice).

List of R. lupini strains having same efficiency on two lupin species (in effectiveness classes).

\begin{tabular}{|c|c|c|c|c|c|c|}
\hline \multirow[t]{2}{*}{ Comparaisons } & \multirow{2}{*}{$\begin{array}{c}\text { Nombre de } \\
\text { souches }\end{array}$} & \multicolumn{5}{|c|}{ Classes d'efficacité } \\
\hline & & $0-40$ & $40-80$ & & $80-12$ & \\
\hline L. albus-L. luteus & 35 & $L L 90\left(^{*}\right) L B 86$ & LL92 LL101 & $\begin{array}{l}\text { LL13 } \\
L B 29 \\
\text { LL96 } \\
\text { LL103 } \\
\text { LLH31 }\end{array}$ & $\begin{array}{l}\text { LL19 } \\
\text { LL87 } \\
\text { LL99 } \\
\text { LL1051 }\end{array}$ & $\begin{array}{l}\text { LB28 } \\
\text { LL94 } \\
\text { LL102 } \\
\text { LL107 }\end{array}$ \\
\hline L. albus-L. mutabilis & 24 & $\begin{array}{l}L L 90 \text { LL15 } \\
(L B 86)(* *)\end{array}$ & & $\begin{array}{l}\text { LL13 } \\
\text { LL82 } \\
\text { LL87 } \\
\text { LLH8 }\end{array}$ & $\begin{array}{l}\text { LB29 } \\
\text { LB84 } \\
\text { LL88 } \\
\text { LLH26 }\end{array}$ & $\begin{array}{l}\text { LB822 } \\
\text { LL84 } \\
\text { LL1051 } \\
\text { LLH40 }\end{array}$ \\
\hline L. luteus-L. mutabilis & 18 & $L L 90(L B 86)(* *)$ & & $\begin{array}{l}\text { LL13 } \\
\text { LL1051 }\end{array}$ & $L B 29$ & $L L 87$ \\
\hline
\end{tabular}

(*) $L L$ ou $L B$ : souches stables dans les trois comparaisons.

(**) Compte tenu des résultats de l'essai au champ.

\section{TABLEAU 8}

Efficacité fixatrice de 6 souches de R. lupini associées à 8 cultivars de L. albus (poids sec des parties aériennes en p. 100 de la moyenne des souches $L L 13$ et LB29; moyenne de 4 répétitions).

Efficiency of $6 \mathrm{R}$. lupini strains with $8 \mathrm{~L}$. albus cultivars (shoot dry weight as \% of the average of strains LL13 and LB29; mean of 4 replicates).

\begin{tabular}{|c|c|c|c|c|c|c|c|c|}
\hline Souches & Multolupa & Blanca & Kalina & $\begin{array}{r}\text { Cultivar de } \\
\text { La57 }\end{array}$ & $\begin{array}{r}\text { Lupinus albus } \\
\text { Lublanc }\end{array}$ & Kali & Neuland & Ultra \\
\hline $\begin{array}{l}L B 29 \\
L L 13 \\
\text { LB28 } \\
\text { LB84 } \\
\text { LL82 } \\
\text { LL15 }\end{array}$ & $\begin{array}{r}96 a \\
104 a \\
87 a \\
89 a \\
98 a \\
79 a\end{array}$ & $\begin{array}{r}100 \mathrm{a} \\
100 \mathrm{a} \\
100 \mathrm{a} \\
92 \mathrm{a} \\
90 \mathrm{a} \\
11 \mathrm{~b}\end{array}$ & $\begin{array}{r}115 \mathrm{a} \\
85 \mathrm{a} \\
98 \mathrm{a} \\
89 \mathrm{a} \\
89 \mathrm{a} \\
32 \mathrm{~b}\end{array}$ & $\begin{array}{r}97 \mathrm{a} \\
103 \mathrm{a} \\
102 \mathrm{a} \\
101 \mathrm{a} \\
80 \mathrm{a} \\
17 \mathrm{~b}\end{array}$ & $\begin{array}{r}99 \mathrm{a} \\
101 \mathrm{a} \\
93 \mathrm{a} \\
90 \mathrm{a} \\
96 \mathrm{a} \\
18 \mathrm{~b}\end{array}$ & $\begin{array}{l}109 \mathrm{a} \\
91 \mathrm{ab} \\
76 \mathrm{~b} \\
85 \mathrm{ab} \\
71 \mathrm{~b} \\
12 \mathrm{c}\end{array}$ & $\begin{array}{c}96 \mathrm{~b} \\
104 \mathrm{ab} \\
118 \mathrm{a} \\
105 \mathrm{ab} \\
102 \mathrm{ab} \\
17 \mathrm{c}\end{array}$ & $\begin{array}{c}98 \mathrm{a} \\
102 \mathrm{a} \\
104 \mathrm{a} \\
66 \mathrm{~b} \\
81 \mathrm{ab} \\
18 \mathrm{c}\end{array}$ \\
\hline $\begin{array}{c}\text { Moyenne des } \\
\text { souches } \\
\text { LL13-LB29 } \\
\text { (g par port) } \\
\text { CV }\end{array}$ & $21,8 \%$ & $17,2 \%$ & $22,7 \%$ & 47,1 & $15,5 \%$ & $22,6 \%$ & $14.2 \%$ & 53,3 \\
\hline
\end{tabular}

vars de L. albus : « Blanca », « Kali », « Kalina », « La57 », « Lublanc », « Multolupa », « Neuland» et « Ultra». Les résultats sont regroupés dans le tableau 8.

Les souches ne se comportent pas de la même façon sur chaque cultivar, ce que confirme une interaction cultivar $\times$ souche, significative au seuil de probabilité de 1 p. 100. Ainsi. sur "Multolupa », il n'y a pas d'effet souche: les 6 souches sont d'efficacité comparable; sur 4 cultivars ( «a57 », « Blanca », « Lublanc » et « Kalina »), 5 souches (LL13, LB28, LB29, LL82 et LB84) sont bonnes fixatrices, et une, LL15, peu fixatrice ; sur 3 autres cultivars (« Neuland », «Ultra » et « Kali »), la souche LL15 est peu fixatrice et les 5 autres souches sont d'efficacité variable.

Parmi les 6 scuches essayées, LL13 et LB29 peuvent être considérées comme bonnes fixatrices sur les 8 cultivars de L. albus, la souche $\mathrm{LB} 28$ se révélant cependant la meilleure sur le cultivar «Neuland».

\section{DISCUSSION ET CONCLUSIONS}

Sur une espèce donnée de lupin, les résultats expérimentaux montrent que l'efficacité fixatrice de $R$. lupini est un caractère variable d'une souche à l'autre, mais stable pour une souche déterminée ; à l'exception des souches LL96 et LL82 (dans les essais I et II, leur efficacité moyenne peut être attribuée à une inoculation trop faible), les souches communes à plusieurs essais réalisent des performances comparables d'un essai à l'autre, qu'elles soient d'efficacité bonne (LL13, LB29 sur les 3 espèces), moyenne (LB28 sur L. mutabilis) ou faible (LL15 sur L. albus, LL82 sur L. luteus ou LLH31 sur L. mutabilis).

Mais cette efficacité fixatrice ne s'exprime pas toujours avec la même intensité sur les 3 espèces de lupin utilisées pour cette étude : ainsi dans l'essai IV (tabl. 4), 2 souches (LL13 et LB29) sur les 11 essayées conservent la même. 
efficacité sur les 3 espèces ; pour l'ensemble des résultats (tabl. 7) la proportion de ce type de souches passe à un tiers, et 22 p. 100 seulement (LL13, LB29, LL87 et LL1051) ont une bonne efficacité fixatrice.

Au sein d'une espèce (L.albus), l'efficacité fixatrice d'une souche de $R$. lupini est influencée par le cultivar auquel elle est associée ; parmi les souches testées, aucune souche de bonne efficacité ne devient peu fixatrice, mais certaines (LB28 et LB29), équivalentes sur la plupart des cultivars, diffèrent significativement sur «Neuland» et «Kali ».

Les interactions espèces $\times$ souches sont des phénomènes fréquents dans les groupes d'inoculation croisée; elles permettent de décrire des sous-groupes en classant les Rhizobium ou les espèces végétales selon leurs réactions symbiotiques : ainsi BROCKWELL \& HELY (1966) divisent un lot de $R$. meliloti en 9 sous-groupes; les travaux de SAUBERT \& SCHEFFLER (1967), complétant les observations de VINCENT (1954), divisent le groupe des trèfles en 3 sousgroupes. Nos résultats permettent d'envisager des subdivisions dans le groupe $R$. lupini, mais ils ne portent que sur 3 des 150 espèces du genre Lupinus (ALLEN \& ALLEN, 1981) et JENSEN (1967) a décrit des relations entre le groupe $R$. lupini et d'autres groupes d'inoculation.

Les interactions cultivar $\times$ souche que nous trouvons dans l'espèce $L$. albus se rencontrent également dans d'autres groupes : luzerne (GASSER et al., 1972 ; MILLER \& SIROIS, 1982), soja (BALASUNDARAM et al., 1972), trèfle blanc (MYTTON, 1975).

L'existence de ces interactions dans le groupe des $R$. lupini permet de proposer le schéma de sélection de souches suivant : début du tri sur L. luteus, la sélection se poursuivant ensuite sur $L$. mutabilis et $L$. albus mais en remplaçant le cultivar «Blanca » par « Kali » ou «Ultra ».

L'efficacité fixatrice mesurée en serre se conserve au champ pour 4 souches de $R$. lupini essayées sur les 3 espèces de lupin, que les souches soient de bonne ou de faible efficacité fixatrice ; parallèlement à une augmentation de rendement les souches ayant une bonne efficacité fixatrice entraînent presque toujours une augmentation de la teneur en protéines des graines, amplifiant ainsi l'effet de l'inoculation sur la production de protéines ; un tel résultat est déjà signalé par BONNIER et al. (1964) sur L. luteus. Mais certaines caractéristiques du sol peuvent modifier l'expression de l'efficacité des souches en agissant sur la nodulation (LANGE \& PARKER, 1960); le travail de sélection doit nécessairement se terminer au champ.

Lorsque le sol contient $R$. lupini, il est peu probable que L. albus rencontre des populations peu fixatrices; les risques sont plus élevés pour $L$. mutabilis et surtout pour $L$. luteus. Le remplacement de telles souches par inoculation nécessiterait au préalable de considérer la compétitivité relative de la souche efficace apportée par rapport à celles du sol, ajoutant ainsi une nouvelle étape aux opérations de sélection.

Reçu le 11 janvier 1983. Accepté le 21 avril 1983.

\section{REMERCIEMENTS}

Nous tenons à remercier nos collègues de la Station d'Amélioration des plantes et du domaine de l'I.N.R.A. de Dijon-Epoisses pour la fourniture des semences et la conduite de l'essai au champ ainsi que M. SIXDENIER qui a réalisé les analyses de protéines.

\section{RÉFÉRENCES BIBLIOGRAPHIQUES}

Allen N. O., Allen K. A., 1981. The Leguminosae. A source book of characteristics, uses and nodulation. The University of Wisconsin Press, Madison, $812 \mathrm{p}$.

Balasundaram V. R., Iswaran V., Sundara Rao W. V. B., 1972. Interactions between soybean (Glycine max (L.) Merr.) genotypes and different isolates of Rhizobium japonicum. Indian J. agric. Sci., 42, 387-389.

Bergersen F. J., 1961. The growth of Rhizobium in synthetic media. Aust. J. biol. Sci., 14, 349-360.

Bonnier C., Laloux L., Lebrun F., Weickmans L., 1964. Inoculation bactérienne des graines de lupin jaune doux (Lupinus luteus). Bull. Inst. agron. Sta. Rech. Gembloux, XXXII, 163-170.

Brockwell J., Hely F. W., 1966. Symbiotic characteristics of Rhizobium meliloti : an appraisal of the systematic treatment of nodulation and nitrogen fixation interactions between hosts and Rhizobia of diverses origins. Aust. J. agric. Res., 17, 885-899.

Caradus J. R., Silvester W. B., 1979. A comparative study of strains of Rhizobium lupini on Lupinus arboreus L. N.Z. J. agric. Res., 22, 329-333.

Gasser H., Guy P., Obaton M., Sikora I., 1972. Efficiency of Rhizobium meliloti strains and their effects on alfalfa cultivars. Can. J. Plant Sci., 52, 441-448.

Gladstone J. S., 1970. Lupins as crop plants. Field Crop Abstr., 23, 124-148.

Graham P. H., 1976. Identification and classification of root nodule bacteria, 99-112. In : Symbiotic nitrogen fixation in plants, Nutman P.S. (ed.), Cambridge University Press, Cambridge, 584 p.

Jensen H. L., 1967. Mutual host plant relationships in two groups of legume root nodule bacteria (Rhizobium spp.). Arch Mikrobiol., 59, 174-179.

Lagacherie B., Ginies P., Amarger N., 1976. L'inoculation du lupin. Essais préliminaires, C.R. Acad. Agric. Fr., 62, 987-994.

Lagacherie B., Hugot R., Amarger N., 1977. Sélection de souches de Rhizobium japonicum d'après leur compétitivité pour l'infection. Ann. agron., 28, 379-389.

Lange R. T., Parker C. A., 1960. The symbiotic performance of lupin bacteria under glasshouse and field conditions. Plant and Soil, 13, $137-146$

Lenoble M., Picard J., 1980. Lupin : où en est-on... Cultivar, 127, 38-40.

Miller R. W., Sirois J. C., 1982. Relative efficacy of different alfalfa cultivar, Rhizobium meliloti strain combinations for symbiotic nitrogen fixation. Appl. environ. Microbiol., 43, 764-768.

Mytton L. R., 1975. Plant genotype $\times$ Rhizobium strain interactions in white clover. Ann. appl. Biol., 80, 103-107.

Saubert S., Scheffler J. G., 1967. Strain variation and host specificity of Rhizobium. II. Host specificity of Rhizobium trifolii on European clovers. S. Afr. J. agric. Sci., 10, 85-94.

Vincent J. M., 1954. The root-nodule bacteria of pasture legumes. Proc. Linn. Soc. N.S.W., 79, 4-32. 